\title{
Novel stability and passivity analysis for three types of nonlinear LRC circuits
}

\author{
Muzaffer Ateş ${ }^{a^{*}}$ and Nezir Kadah ${ }^{b}$ \\ ${ }^{a}$ Departments of Electrical-Electronics Engineering, University of Van Yuzuncu Yil, Turkey \\ ${ }^{b}$ Departments of Electrical-Electronics Engineering, Adana Alparslan Turkes Science and Technology \\ University, Turkey \\ mates@yyu.edu.tr,nkadah@atu.edu.tr
}

\section{ARTICLE INFO}

\section{Article History:}

Received 08 January 2021

Accepted 09 May 2021

Available 31 July 2021

\section{Keywords:}

Lyapunov stability

Nonlinear systems

Nonlinear LRC circuits

Passivity

Gronwall's inequality

AMS Classification 2010:

34D23; 34D20; $34 C 23$

\begin{abstract}
In this paper, the global asymptotic stability and strong passivity of three types of nonlinear $L R C$ circuits are investigated by utilizing the Lyapunov's direct method. The stability conditions are obtained by constructing appropriate energy (or Lyapunov) function, which demonstrates the practical application of the Lyapunov theory with a clear perspective. Many specialists construct Lyapunov functions by using some properties of the functions with much trial and errors or for a system they choose candidate Lyapunov functions. So, for a given system the Lyapunov function is not unique. But we insist that the Lyapunov (energy) function is unique for a given physical system. Thus, this study clarifies Lyapunov stability with suitable tools and also improves some previous studies. Our approach is constructing energy function for a given nonlinear system that based on the power-energy relationship of the system. Hence for a dynamical system, the derivative of the Lyapunov function is equal to the negative value of the dissipative power in the system. These aspects have not been addressed in the literature. This paper is an attempt towards filling this gap. The provided results are central importance for the stability analysis of nonlinear systems. Some simulation results are also given successfully that verify the theoretical predictions.

(cc) $\mathrm{Br}$
\end{abstract}

\section{Introduction}

In history, modeling and stability analysis of nonlinear systems are the most important and popular problems in control theory. Since almost all systems are nonlinear in nature [1], a number of promising studies have been analyzed in the literature. Many researchers as Lagrange, Hamilton, Poincare and Lyapunov are focused on the modelling problem to analyze the dynamic behavior of systems [1, 2]. The aforementioned methods are based on the energy utilization of the related systems. However, since all systems are not in linear forms, certain mathematical solutions are not available to solve these issues. Furthermore, closed-form expressions for the solutions of the linear systems are not possible to solve nonlinear systems. Nevertheless, it is important to be able to make some assumptions about the conduct of a nonlinear system called qualitative analysis.

The stability of the equilibrium point was first examined by Lagrange; however, the Lagrange principle was only suitable for the Lagrange systems (conservative systems) [1], but engineering systems usually have damping [3]. Then, the stability theory of motion derived from the concepts of Lagrange's principle and Poincare's regular solution (Lyapunov stable motion) was developed by Lyapunov [2]. Hamiltonian and Lagrangian systems comply with conservative systems (exact differential equations), but Lyapunov stability theory can be applicable to arbitrary differential equations. Thus, the Lyapunov direct method is 
the most common and efficient tool for stability analysis [4 7]. In this context, many elegant studies on the qualitative behavior of systems can be recorded in the literature. Most experiments are carried out on the axiomatization of the stability principle. The problem of stability of the solar system attracted a great deal of early interest. Then, Lyapunov used his second (direct) method that there is no need to solve the differential equations explicitly to investigate the stability of the given systems. The Lyapunov's direct method is still recognized as an effective tool to study the stability theory of dynamical systems such as: the global asymptotic stability of the electrical RLC circuit [8], neural networks with time varying delays 9 10], power systems analysis [11], robot manipulators [12, dissipativity analysis of discretetime neural networks [13], global robust passivity analysis 14, dissipativity and passivity analysis of neural networks 15. This method is the best way to determine the asymptotic stability or asymptotic controllability of nonlinear systems. The central notion is that the energy of the system diminishes along suitably chosen paths, such that the system attains a minimal energy configuration at the invariant equilibrium. Here, this result has been presented both mathematically and through simulation.

Lyapunov theory is based on the Torricelli principle [16]. Therefore, the storage energy of the dynamic system decreases over time along the trajectories of the system. So, the direct method provides the opportunity to examine the stability of the equilibrium points with minimum energy. This meaning (diminishing of energy) tends us to the passivity of the systems. Passivity, which is the basic feature of the dynamic systems theory [17 20], can now be debated. LRC circuits, viscoelastic systems and thermodynamic systems are typical examples of dissipative systems with the external sources. The terminology of dissipativity is a generalization of the concept of passivity [21]. Apparently, a dissipative system is not a conservative system. The main point of passivity theory is that the systems are internally stable [22, 23]. Storage functions are bounded [21], and this result has been proved mathematically in the proof of Theorem 4. Thus, some new passivity results with Gronwall's inequality [3] can be shown to define the strict passivity or boundedness of the systems involved.

Natural (real) energy functions of the dynamic systems empower the Lyapunov's direct process implementations more than the Lyapunov candidate functions. Thus, each energy function used in this study is constructed from the physical meaning of the given system and its time derivative (directional) is equal to the negative value of the dissipated power in the system. For example, for any unforced dissipative system, the time derivative of the energy (Lyapunov) function $E(t)$ along the system orbits gives

$$
V^{\prime}(t)=-\sum_{i=1}^{n} R_{i} I_{i}^{2},
$$

where $R_{i}$ is the damping term (or resistance) and $I_{i}$ is the velocity (or current) of the $i$ th component of the system.

The above arguments are not clear in the related literature. Hence, many specialists chose candidate Lyapunov functions or consider some Lyapunov functions or construct Lyapunov functions with much trial and error for their systems [1, 11, 24, 25 without any physical meaning. Generally, these tools make Lyapunov stability very complex (see 24,25$])$. Because, still there is an idea in the literature, constructing Lyapunov functions for nonlinear systems is a difficult task [26, 27]. But, for the first and second order ordinary differential equations we highly simplified Lyapunov stability theory with $L R C$ circuit systems. Hence, the interested knows how to construct the energy (Lyapunov) function and checks the result of the time derivative (directional) of the energy function with (1). This approach also improves some well-known studies. These improvements will take place in section 4 . In addition, [6 does not involve the passivity analysis, the notion of power -energy relationship constructing Lyapunov functions, and equation (1) and its implications. The present work includes these and some improvements.

The rest of this paper is organized as follows. Section 2 presents some definitions and auxiliary results. Section 3 deals with the main results. Section 4 deals with discussion. Section 5 closes the paper with a short conclusion.

\section{Preliminaries}

A commonly used model for an autonomous nonlinear system is

$$
x^{\prime}(t)=f(x(t), u(t)), \quad x(0)=x_{0}, \forall t \geq 0,
$$

where $t \in \Re_{+}\left(\Re_{+}=[0, \infty)\right)$ denotes time, $x \in \Re^{n}$ denotes the state of the system, while $u \in \Re^{m}$ is called the input or the control function. However, 
$f: \Re^{n} \times \Re^{m} \rightarrow \Re^{n}$ satisfies Lipschitz condition. The state vector $x(t) \in D$ in which $D \subseteq \Re^{n}$ is a domain that contains the origin $x=0$. We assume that (2) is well posed, that is, there exists a unique solution $x:[0, \infty) \rightarrow \Re^{n}$ for every initial data $x(0)=x_{0} \in \Re^{n}$, and $x$ depends continuously on $x_{0}$ according to the normed topology on $\Re^{n}$. Let $f(0,0)=0, f(x, 0) \neq 0$ for $x \neq 0$, and $\|$.$\| is$ the Euclidean norm on $\Re^{n}$. Further, assume that $u$ is an admissible real valued input function so that

$$
\sum_{i=1}^{m} \int_{0}^{t} u_{i}(t) d t \leq K<\infty, \quad \forall t>0,
$$

where $K$ is a positive constant. A state $\bar{x} \in \Re^{n}$ is an equilibrium of (2) if $f(\bar{x}, 0)=0$. A system or machine attains its minimum of energy at the equilibrium points.

We shall now need some basic definitions on the properties of the Lyapunov functions.

Definition 1. ([1]) A function $\alpha\left(\Re^{+}, \Re^{+}\right)$is of class $\kappa$ if it is continuous on $[0, \infty)$, monotonically increasing, and $\alpha(0)=0$. A class $\kappa$ function $\alpha(r)$ belongs to class $\kappa_{\infty}$ if $\alpha(r) \rightarrow \infty$ as $r \rightarrow \infty$.

Definition 2. ( [1]) A function $E(x) \in C^{1}\left(\Re^{+} \times\right.$ $\left.\Re^{n}, \Re^{+}\right)$is said to be positive definite, decrescent and radially unbounded function if there exist functions $\alpha$ and $\beta$ of class $\kappa$ are such that

(i) $\alpha(\|x\|) \leq E(x) \leq \beta(\|x\|), \quad \forall x \in \Re^{n}$,

(ii) $E^{\prime}(x(t)) \leq 0$,

(iii) $\alpha(\|x\|) \rightarrow \infty$ as $\|x\| \rightarrow \infty$,

(iv) Furthermore, assume that the set $S=\{x \in$ $\left.\Re^{n}: E^{\prime}(x)=0\right\}$, contains no invariant set other than the set $\{0\}$.

Now, to motivate the definitions of passivity we can use electrical circuits. Inflow power of a simple resistive system is always nonnegative with the voltage $u(t)$ as input and the current $y(t)$ as output, that is, if $u y \geq 0$ for all $u, y \in \Re$, and for a multiport network we have $u^{T} y=\sum_{i=1}^{m} u_{i} y_{i} \geq 0$. Therefore, for general nonlinear systems we can state some new passivity properties. These new properties are of interest in circuit and control theory [24] and has applications in mathematical control theory with Gronwall's inequality [3].

Definition 3. ( [24]) System (2) is passive if there exists a positive definite function $E(t)$ is such that

$$
\text { (i) } \quad E^{\prime}(t) \leq r(u, y)
$$

Moreover, it is lossless if

$$
\text { (ii) } \quad E^{\prime}(t)=r(u, y)
$$

and strictly passive if

$$
\text { (iii) } \quad E^{\prime}(t)+\Psi(x, y) \leq r(u, y)
$$

for some positive definite function $\Psi$, where $y=$ $x^{\prime}$, and $r(t)=r(u(t), y(t))=\sum_{i=1}^{m} u_{i} y_{i}$ is the supply rate function of (2) defined on $\Re^{m} \times \Re^{n}$, and satisfies

$$
\int_{0}^{t}|r(s)| d s<\infty
$$

for all $t \geq 0$, with $r(0, y)=0$.

Lemma 1. If system (1) is passive with an energy-like function $E$, then the origin of $x^{\prime}=$ $f(x, 0)$ is stable.

Proof. See 24].

\section{Main results}

The matter under discussion is the stability of the origin $(0,0)$ and the passivity of the following nonlinear resistive, inductive, and capacitive $L R C$ circuits for one input variable $(m=1)$ and two state variables $(n=2)$. In this work, the inputs of the systems (circuits) are bounded and admissible continuous functions in $t$. In addition, a nonlinear resistor can be both current controlled $(R(i))$ and voltage controlled $(R(v))$ element. A nonlinear inductor is current controlled $(L(i))$ element while a nonlinear capacitor is said to be voltage controlled $(C(v))$ element. The internal resistance of a current source is infinite while that of a voltage source is finite and especially chosen to be small.

\subsection{Nonlinear resistor}

In the following circuit [28] (Figure 11) there is a nonlinear resistive element which is specified by $i_{2}=f\left(v_{2}\right)$ and the remaining components $R_{1}, R_{2}$, $L, C$ are positive scalars. 


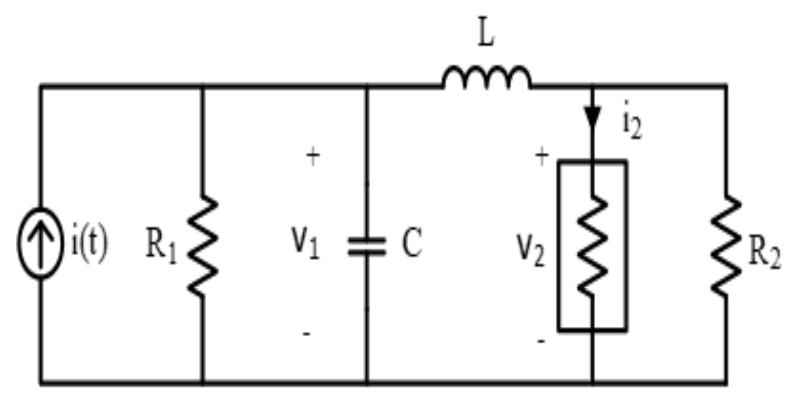

Figure 1. $L R C$ circuit with nonlinear resistive element.

Theorem 1. The nonlinear element of the timeinvariant circuit shown in Figure 1 is specified by the relation $i_{2}=f\left(v_{2}\right)$. $v_{1}$ and $v_{2}$ are the state variables of the circuit. Then, the solution $v(t)=0$ to the system.

$$
\left\{\begin{array}{l}
v_{1}^{\prime}=\frac{1}{C}\left[i(t)-\frac{v_{1}}{R_{1}}-f\left(v_{2}\right)-\frac{v_{2}}{R_{2}}\right] \\
v_{2}^{\prime}=\left[f^{\prime}\left(v_{2}\right)+\frac{1}{R_{2}}\right]^{-1}\left[\frac{v_{1}-v_{2}}{L}\right]
\end{array}\right.
$$

with $i(t)=0$, is globally asymptotically stable and the circuit will be lossless at infinity if

(i) $v_{1}>v_{2}$,

(ii) $v_{2} f\left(v_{2}\right) \geq 0$,

(iii) $f(0)=0$.

Proof. First, let write down the state equations of the above circuit:

$$
\left\{\begin{array}{l}
i(t)=\frac{v_{1}}{R_{1}}+C v_{1}^{\prime}+f\left(v_{2}\right)+\frac{v_{2}}{R_{2}}, \\
v_{1}-v_{2}=L \frac{d}{d t}\left[f\left(v_{2}\right)+\frac{v_{2}}{R_{2}}\right]=L\left[f^{\prime}\left(v_{2}\right) v_{2}^{\prime}+\frac{v_{2}^{\prime}}{R_{2}}\right] .
\end{array}\right.
$$

Then after some arrangement we obtain system (4). The natural energy function $E_{1}(t)=$ $E_{1}\left(v_{1}, v_{2}\right)$ from the storage elements (capacitor and inductor) of this circuit is

$$
E_{1}(t)=\frac{1}{2} C v_{1}^{2}+\frac{1}{2} L\left[f\left(v_{2}\right)+\frac{v_{2}}{R_{2}}\right]^{2} .
$$

The energy function $\left(E_{1}: \Re^{2} \rightarrow \Re^{+}\right)$satisfies

(i) $E_{1}(0)=0$,

(ii) $E_{1}(v)>0, \quad \forall v \in \Re^{2}-\{0\}$.

$E_{1}$ is confirmed by the hypothesis (i) of Definition 2. Thus, $E_{1}$ is a positive definite function. Then, we write

$$
E_{1}(t) \geq \frac{1}{2} C v_{1}^{2} \equiv \alpha\left(\left\|v_{1}\right\|\right)
$$

The derivative of the Lyapunov function along the trajectories of system (4) gives

$$
E_{1}^{\prime}(t)=C v_{1} v_{1}^{\prime}+L\left[f\left(v_{2}\right)+\frac{v_{2}}{R_{2}}\right]\left[f^{\prime}\left(v_{2}\right)+\frac{1}{R_{2}}\right] v_{2}^{\prime},
$$

By using system (4), we have

$$
\begin{array}{r}
E_{1}^{\prime}(t)=i(t) v_{1}-\frac{v_{1}^{2}}{R_{1}}-f\left(v_{2}\right) v_{1}-\frac{v_{1} v_{2}}{R_{2}} \\
+\left[f\left(v_{2}\right)+\frac{v_{2}}{R_{2}}\right]\left(v_{1}-v_{2}\right), \\
E_{1}^{\prime}(t)=i(t) v_{1}-\frac{v_{1}^{2}}{R_{1}}-f\left(v_{2}\right) v_{1}-\frac{v_{1} v_{2}}{R_{2}} \\
+f\left(v_{2}\right) v_{1}+\frac{v_{1} v_{2}}{R_{2}}-f\left(v_{2}\right) v_{2}-\frac{v_{2}^{2}}{R_{2}} \\
E_{1}^{\prime}(t)=i(t) v_{1}-\frac{v_{1}^{2}}{R_{1}}-f\left(v_{2}\right) v_{2}-\frac{v_{2}^{2}}{R_{2}} .
\end{array}
$$

For $i(t)=0$, it follows that

$$
\begin{aligned}
E_{1}^{\prime}(t) & =-\frac{v_{1}^{2}}{R_{1}}+\frac{v_{2}^{2}}{R_{2}}-v_{2} f\left(v_{2}\right) \\
& =-R_{1} I_{R_{1}}^{2}-R_{2} I_{R_{2}}^{2}-R_{N L} i_{2}^{2}
\end{aligned}
$$

where $R_{N L}$ represents the resistance value of the nonlinear element. $E_{1}^{\prime}$ is verified by (1). The application of Theorem 1 shows that: $E_{1}^{\prime} \leq 0$ on $\Re^{2}$, $E_{1}(\infty)=0$ and $E_{1}(v) \rightarrow \infty$ as $\|v\| \rightarrow \infty$. Hence, all the motions of (4) are bounded (as illustrated in Figure 2a, 2b). The set $S$ where $E_{1}^{\prime}=0$ is $\{0,0\}$. This implies that $\{0,0\}$ is the only invariant subset of $\mathrm{S}$, and the zero solution or equilibrium solution of (4) is globally asymptotically stable. It can be seen that (4) is a lossless system at infinity due to $v(t)=0$. Hence, the system is zero-state observable.

\subsection{Nonlinear inductor}

The circuit shown in Figure 324 contains a nonlinear inductive element and is driven by a timedependent current source $i_{s}(t)$. Suppose the nonlinear inductor is a Josephson junction described by $i_{L}=a \phi(t)+b \phi^{3}(t)$, where $\phi$ is the magnetic flux of the inductor, $a, b>0$ are positive constants. The remaining elements $R$ and $C$ are linear and have positive real values. 


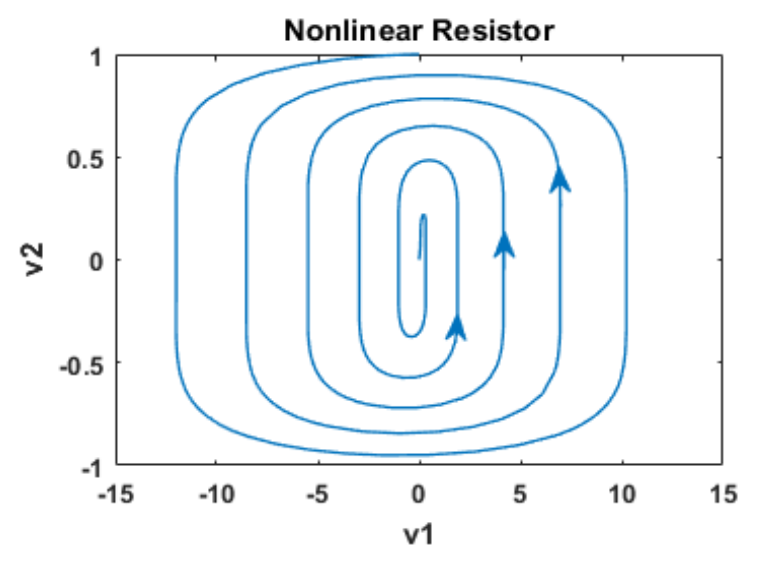

(a) Phase plane plot of system (4) with $L=\frac{1}{15} H$

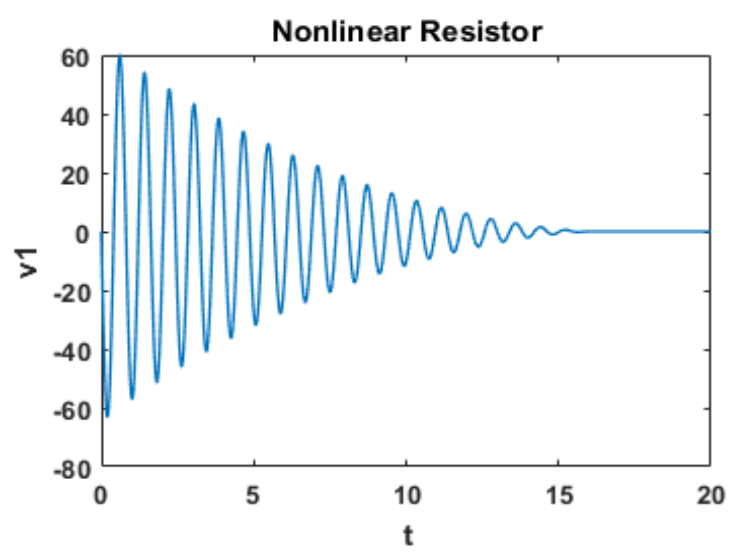

(b) Solution of system (4) with $L=\frac{4}{3} H$

$$
\left[R_{1}=R_{2}=1 k \Omega, C=10 \mathrm{mF}, f\left(v_{2}\right)=5 v_{2}^{3}\right]
$$

Figure 2. The motions of system (4).

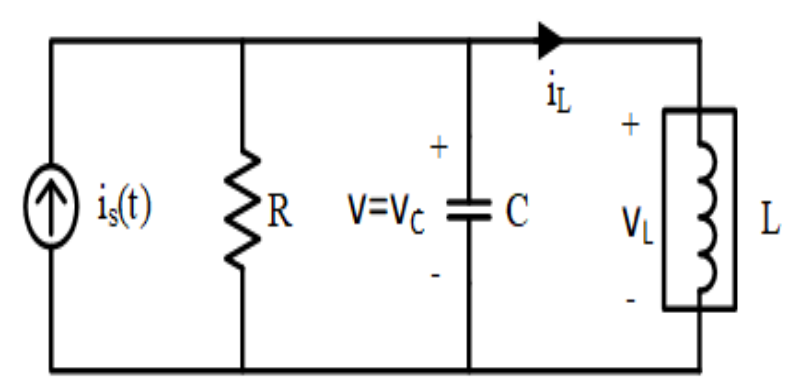

Figure 3. $L R C$ circuit with nonlinear inductive element.

Theorem 2. Let $\phi$ and $v\left(=v_{L}\right)$ be the state variables of the above circuit (Figure 3) with $i_{L}=$ $a \phi(t)+b \phi^{3}(t)$. Then, the solution $(\phi(t), v(t))=$ $(0,0)$ to the system

$$
\left\{\begin{array}{l}
\phi^{\prime}=\frac{v}{L\left(a+3 b \phi^{2}\right)} \\
v^{\prime}=\frac{1}{C}\left[i_{s}(t)-\frac{v}{R}-a \phi-b \phi^{3}\right]
\end{array}\right.
$$

with $i_{s}(t)=0$ is globally asymptotically stable or the circuit is lossless at infinity.

Proof. First, let write down the node and $v$ equations of the above circuit:

$$
\left\{\begin{array}{l}
v=L \frac{d}{d t}\left(a \phi+b \phi^{3}\right) \\
i_{s}(t)=\frac{v}{R}+C v^{\prime}+a \phi+b \phi^{3}
\end{array}\right.
$$

Then after some rearrangement we obtain system (6).

The native energy function for this circuit is

$$
E_{2}(t)=E_{2}(\phi, v)=\frac{1}{2} L\left(a \phi+b \phi^{3}\right)^{2}+\frac{1}{2} C v^{2}
$$

The energy function $\left(E_{2}: \Re^{2} \rightarrow \Re^{+}\right)$satisfies

(i) $E_{2}(0)=0$,

(ii) $E_{2}(\phi, v)>0, \forall(\phi, v) \in \Re^{2}-\left\{\sqrt{\phi^{2}+v^{2}} \neq 0\right\}$.

$E_{2}$ is confirmed by the hypothesis (i) of Definition 2. Thus, $E_{2}$ is a positive definite function. Then, we write

$$
E_{2}(t) \geq \frac{1}{2} C v^{2} \equiv a(\|v\|)
$$

The derivative of the energy function $E_{2}$ along the trajectories of system (6) gives

$$
E_{2}^{\prime}(t)=L\left[a \phi+b \phi^{3}\right]\left[a+3 b \phi^{2}\right] \phi^{\prime}+C v v^{\prime}
$$

By using (6), we have

$$
E_{2}^{\prime}(t)=i_{s}(t) v-\frac{1}{2} v^{2}
$$

For $i_{s}(t)=0$, it follows that

$$
E_{2}^{\prime}(t)=-\frac{1}{2} v^{2}=-R I_{R}^{2}
$$

$E_{2}$ is verified by (1). The application of Theorem 2 shows that: $E_{2}^{\prime} \leq 0$ on $\Re^{2}, E_{2}(\infty)=0$ and $E_{2}(\phi, v) \rightarrow \infty$ as $\sqrt{\phi^{2}+v^{2}} \rightarrow \infty$. Hence, all the motions of (6) are bounded (as illustrated in Figure $4 \mathrm{a}, 4 \mathrm{~b}$. The set $S$ where $E_{2}^{\prime}=0$ is $\{\phi, 0\}$ and from (6) this implies that $\{0,0\}$ is the only invariant subset of $\mathrm{S}$, such that the zero solution or equilibrium solution of (6) is globally asymptotically stable. Thus, system (6) with its Lyapunov function satisfies all the assumptions of 


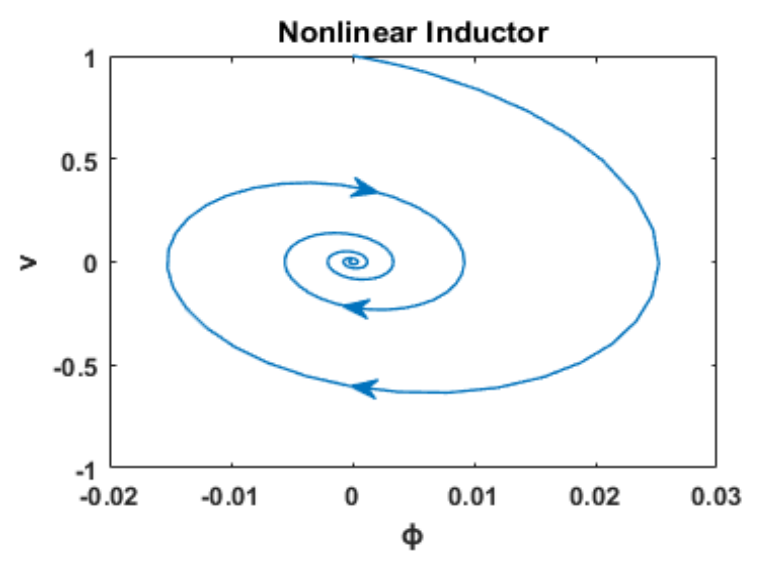

(a) Phase plane plot of system (6)

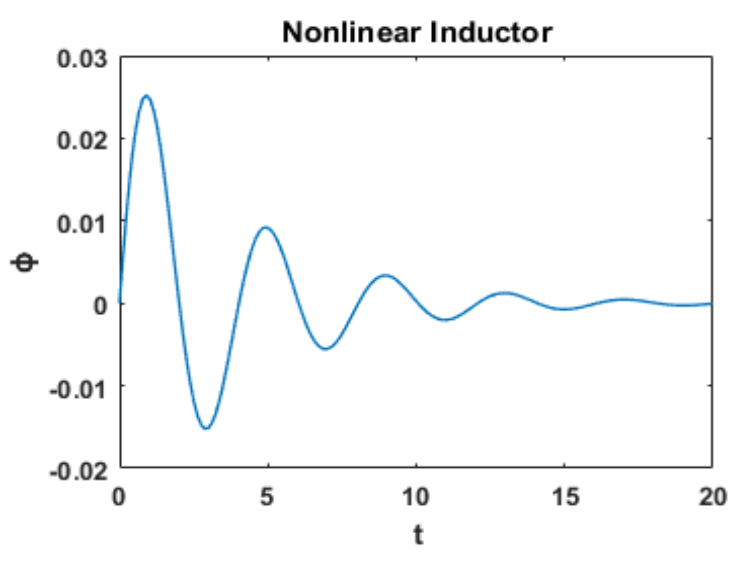

(b) Solution of system (6)

$$
[a=b=1, R=100 \Omega, C=20 m F, L=20 H]
$$

Figure 4. The motions of system (6)

Theorem 2. Therefore, (6) or the related circuit is lossless due to the trivial solution which occurs at infinity.

\subsection{Nonlinear capacitor}

A nonlinear capacitive element is present in the following circuit [29] and its voltage drop is no longer given by $q / C$, but is more accurately described by $\alpha q(t)+\beta q^{3}(t)$, where $\alpha$ and $\beta$ are constants. The remaining elements $L$ and $R$ are positive scalars.

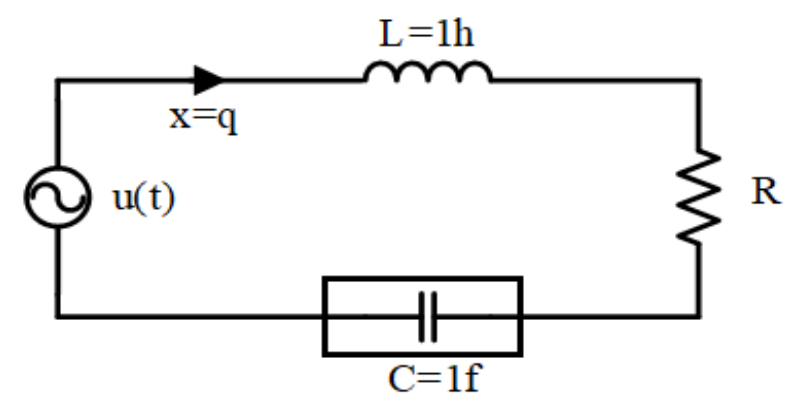

Figure 5. $L R C$ circuit with nonlinear capacitive element.

The above dynamical system generates a differential equation of the form

$$
q^{\prime \prime}+R q^{\prime}+\alpha q+\beta q^{3}=u(t) .
$$

Let, $q=x$ is the flow of the charge, $\alpha$ and $\beta$ are real constants, and $u$ is the applied voltage. Instead of the above equation

$$
\left\{\begin{array}{l}
x^{\prime}=y \\
y^{\prime}=-R y-\alpha x-\beta x^{3}+u,
\end{array}\right.
$$

will be discussed.
Theorem 3. The system (8) is stable if

$$
u=0, \quad \alpha>0, \quad \beta<0
$$

and it is globally asymptotically stable if $\beta<0$ is replaced by $\beta>0$.

Proof. Let $(x(t), y(t))$ be a solution of (8) for $t \geq 0$. In the case of $\beta<0$ : Let $\beta=-c(c>0, \alpha$ constant), the system has the equilibrium point $(0,0)$ and $\left( \pm \sqrt{\alpha c^{-1}}, 0\right)$. In the other case $(\beta>0)$, the system has $(0,0)$ and $\left( \pm \sqrt{\alpha \beta^{-1}}, 0\right)$. Therefore, $(0,0)$ is the only invariant equilibrium point of (8). For the first case we investigate the stability of (8). The storage energy function from power- energy relationship can be constructed in the neighborhood of the equilibrium point $(0,0)$ as

$$
E_{3}(t)=E_{3}(x, y)=\frac{1}{2} y^{2}+\int_{0}^{x}\left(\alpha x+\beta x^{3}\right) d x,
$$

$$
E_{3}(t)=E_{3}(x, y)=\frac{1}{2} y^{2}+\frac{\alpha}{2} x^{2}+\frac{\beta}{4} x^{4} .
$$

The energy function $\left(E_{3}: \Re^{2} \rightarrow \Re^{+}\right)$satisfies

(i) $E_{3}(0)=0$,

(ii) $E_{3}(x, y)>0, \forall(x, y) \in \Re^{2}-\left\{\sqrt{x^{2}+y^{2}} \neq 0\right\}$.

$E_{3}$ is not radially unbounded. But, in the neighborhood of $(0,0), E_{3}$ is positive definite, and we have

$$
E_{3}(t) \geq \frac{1}{2} y^{2}
$$


(9) obeys (i) and (ii) of Definition 2. The derivative of the energy function $E_{3}$ along the trajectories of system (8) gives

$$
E_{3}^{\prime}=y y^{\prime}+\alpha x x^{\prime}+\beta x^{3} x^{\prime}
$$

By using (8), we have

$$
E_{3}^{\prime}=-R y^{2}+u y
$$

For $u(t)=0$, it follows that

$$
E_{3}^{\prime}=-R y^{2}
$$

(10) is verified by (2) and system (8) is stable. . On the other hand, the integration of 100 from 0 to $t(\geq 0)$ gives

$$
E_{3}(x(t), y(t)) \leq E_{3}(x(0), y(0)), \quad t \geq 0 .
$$

That is, $E_{3}$ is a decreasing function along the solution curve $(x(t), y(t))$, and $(0,0)$ is a minimum point of $E_{3}$. The above inequality implies that the motion $(x(t), y(t))$ will stay in the neighborhood of the equilibrium point $(0,0)$ for $t \geq 0$ provided that the initial point $(x(0), y(0))=\left(x_{0}, y_{0}\right)$ is sufficiently near the point $(0,0)$. Hence, the origin is stable. In addition, for $\alpha=1$ and $\beta<0$ we have the following inequalities.

$$
E_{3}(x(t), y(t)) \leq x^{2}(t)+y^{2}(t)
$$

and

$$
E_{3}(x(0), y(0)) \leq x^{2}(0)+y^{2}(0) .
$$

Then, it follows that

$$
\begin{gathered}
x^{2}(t) \leq E_{3}(t) \leq x^{2}+y^{2}, \\
x^{2}(t)+y^{2}(t) \leq x^{2}(0)+y^{2}(0), \quad t \geq 0 .
\end{gathered}
$$

Then, for any given $\epsilon>0$, there is a $\delta>0$. Thus if,

$$
\sqrt{x^{2}(0)+y^{2}(0)}=\sqrt{x_{0}^{2}+y_{0}^{2}}
$$

and

$$
\sqrt{x^{2}(t)+y^{2}(t)}<\epsilon
$$

Then

$$
\left\|x_{0}\right\|<\delta \text { implies that } \quad\|x(t)\|<\epsilon \text {. }
$$

This is precisely the most common definition of stability of a system which has an isolated equilibrium point $(0,0)$.

In the case where $\beta>0: E_{3}^{\prime}(x, y) \leq 0$ at all points $(x, y) \in \Re^{2}$. That is $E_{3}(t)$ is a decreasing function along any motion of (8), $E_{3}(\infty)=0$ and $E_{3}(x) \rightarrow \infty$ as $\|x\| \rightarrow \infty$.Hence, all the solutions of (8) are bounded.The set $S$, where $E_{3}=0$ is $(x, 0)$, and $(0,0)$ is the only invariant subset of $S$. Thus, the application of Theorem 3 shows that the solution $x(t)=0$ to (8) as $t \rightarrow \infty$. Therefore, the system is zero-state observable. This also implies that there is no energy dissipation in the circuit at infinity $(t=\infty)$; that is, the circuit will be lossless at infinity. This explanation is compatible with Figure 6a, 6b, Besides, when the value of $R$ increased, the motion goes to the equilibrium point immediately.

The simulations are verifying our theoretical results. The trajectories in the phase spaces (Figure 2a, 4a, and 6a go to the equilibrium solutions $(x(\infty), y(\infty))=(0,0)$. On the other hand, time series solutions (Figure 2b, 4b, and 6b) approach zero at infinity.

The three strong passivity results or the boundedness of the motions (strict passivity) of (4), (6) and (8) with their input functions are the following.

Theorem 4. Suppose that all the conditions in Theorems 1, 2, and 3 are satisfied and also assume that (3) holds such that

$\max \left\{\int_{0}^{t} i(s) d s, \int_{0}^{t} i_{s}(s) d s, \int_{0}^{t} u(s) d s\right\} \leq K<\infty$, $\forall t>0$,

where $K$ is a positive constant. Then, all the motions of (4), (6) and (8) with their forcing functions are bounded or the systems are strongly passive.

Proof. From the proof of Theorem 1 we have

$$
E_{1}^{\prime}(t)=-\frac{v_{1}^{2}}{R_{1}}-\frac{v_{2}^{2}}{R_{2}}-v_{2} f\left(v_{2}\right)+i v_{1} .
$$

Then 


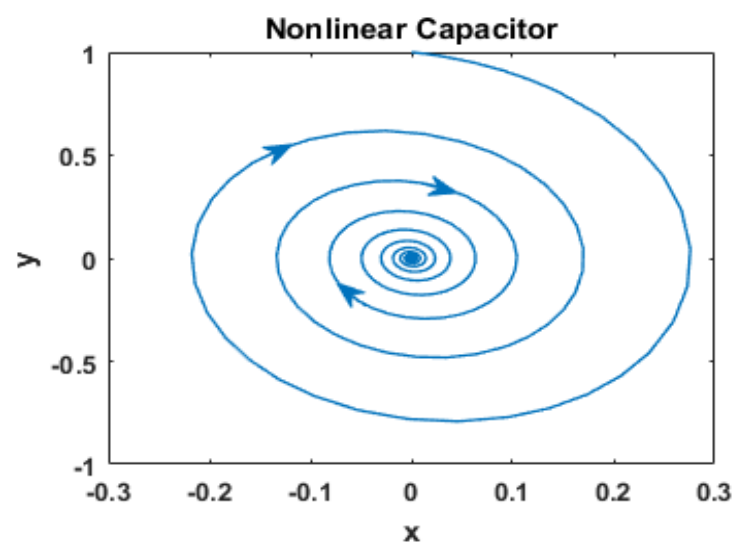

(a) Phase plane plot of system (6)

$$
\lceil R=0.5 \Omega, \alpha=\beta=10]
$$

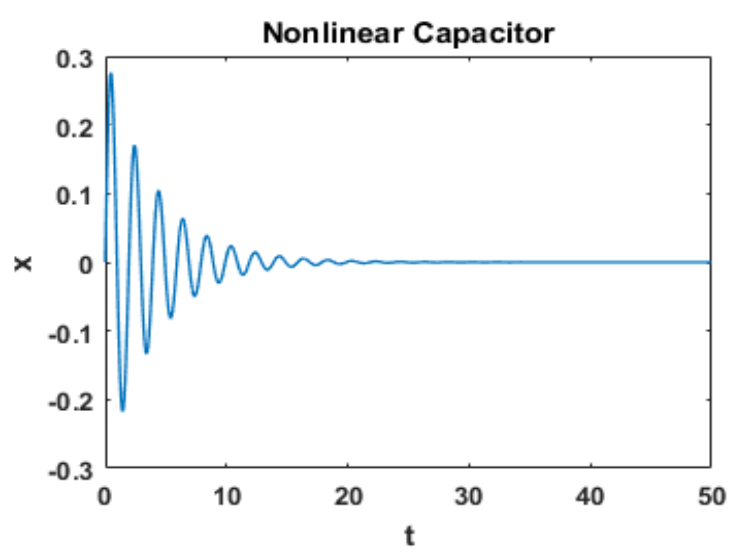

(b) Solution of system 6 ,

Figure 6. The motions of system (6).

$$
E_{1}^{\prime}(t)+\frac{v_{1}^{2}}{R_{1}}+\frac{v_{2}^{2}}{R_{2}} \leq i v_{1}
$$

Hence, by Definition 3, the system (4) is strictly passive.

Furthermore

$$
E_{1}^{\prime}(t) \leq i v_{1} \leq i\left(1+v_{1}^{2}\right),
$$

which also known as the dissipative inequality.

By (5), it follows that

$$
E_{1}^{\prime}(t) \leq i(t)+\frac{2}{C} E_{1}(t) i(t) .
$$

Integrating (11) from 0 to $t(>0)$, and using Theorem 4 , it follows that

$$
E_{1}(t) \leq K+\frac{2}{C} \int_{t_{0}}^{t} E_{1}(s) i(s) d s .
$$

Then, Gronwall's inequality [3] yields

$$
E_{1}(t) \leq K \exp \left(\frac{2 K}{C}\right)
$$

Using the foregoing procedure, the following results are obtained which determine the upper bounds of $E_{2}$ and $E_{3}$, respectively.

$$
E_{2}(t) \leq K \exp \left(\frac{2 K}{C}\right)
$$

and

$$
E_{3}(t) \leq K \exp (2 K)
$$

Finally, the connection between (5) and (12), (7) and (13), and (9) and (14), respectively, give the following results:

$$
\frac{1}{2} C v_{1}^{2} \leq E_{1}(t) \leq K \exp \left(\frac{2 K}{C}\right),
$$

$$
\frac{1}{2} C v^{2} \leq E_{2}(t) \leq K \exp \left(\frac{2 K}{C}\right)
$$

and

$$
\frac{1}{2} y^{2} \leq E_{3}(t) \leq K \exp (2 K)
$$

Thus, the energy functions $E_{1}, E_{2}$, and $E_{3}$ are bounded. This also implies that all the motions of (4), (6), and (8) are bounded in magnitude. Hence, the related systems (or circuits) are strongly passive.

\section{Discussion}

The properties of energy function and its Lie derivative determine the criteria of Lyapunov stability theory. Thus, our natural approach in this paper may be applicable to all physical systems whatever the orders of the systems. Here, we will only improve the stability of some second order systems that relevant to our study. In this connection, the stability of the following differential equations (with their arguments) has been investigated in [25] and [30], respectively,

$$
x^{\prime \prime}+a(t) f\left(x, x^{\prime}\right) x^{\prime}+b(t) g(x)=0
$$




$$
\begin{gathered}
V_{0}=\frac{1}{2} y^{2}+b(t) \int_{0}^{x} g(\xi) d \xi+k, \\
(k>0, \text { constant }), \\
V_{0}^{\prime}=-a(t) f(x, y) y^{2}+b^{\prime}(t) \int_{0}^{x} g(\xi) d \xi ;
\end{gathered}
$$

$$
\begin{aligned}
& x^{\prime}=y, \\
& y^{\prime}=-a(t) f(x, y) y-b(t) g(x) .
\end{aligned}
$$

The natural energy function for (a4) must be

$$
V(t, x, y)=\frac{1}{2} y^{2}+\int_{0}^{x(t)} b(t) g(s) d s .
$$

and

$$
\begin{gathered}
x^{\prime \prime}+x^{\prime}+p(t) g_{1}(x)+q(t) g_{2}(x)=0, \\
V(t, x, y)=\frac{1}{2} y^{2}+p(t) G_{1}(x)+q(t) G_{2}(x),
\end{gathered}
$$

where $G_{i}(x)=\int_{0}^{x} g_{i}(\xi) d \xi \quad(i=1,2)$,

$$
\begin{aligned}
V^{\prime}(t, x, y) & =p^{\prime}(t) G_{1}(x)-p(t) x g_{1}(x) \\
& +q^{\prime}(t) G_{2}(x)-q(t) x g_{2}(x)
\end{aligned}
$$

\section{Remark 1}

There may be some objection regarding to the derivative of Lyapunov functions, but powerenergy relationship shows that this objection is unfounded. For example, consider a series $L R C$ circuit which has $b(t) g(q)$ voltage on a time varying nonlinear capacitor with $q(t)$ charge that flows in the circuit. Let $P_{C}, W_{C}$ be the power and energy of the capacitor. Then, we have the followings:

(i) $P_{C}(t)=b(t) g(q) \frac{d q}{d t}=b(t) g(q) q^{\prime}$,

(ii) $W_{C}(t)=\int_{0}^{q} b(t) g(s) d s, \quad W_{C}(0)=0$

where $d s=q^{\prime}(t) d t$. Then,

(iii) $\frac{d}{d t} W_{C}(t)=b(t) g(q) q^{\prime}$.

Thus, for the construction of energy and power functions that associated with capacitors, we nicely apply the above loop. This may enable us to improve the stability of many systems, because the above algorithm eliminates to take the partial derivative of $W_{C}(t)$.

\section{(A) The natural approach improves the result given in 25] such as:}

(a1) may represent a $L R C$ circuit (dissipative) system with
Since, (a5) has been constructed from the powerenergy relationship of $(a 4)$. The Lie derivative of $(a 5)$ is

$$
V^{\prime}(t, x, y)=-\alpha(t) f(x, y) y^{2} .
$$

(a6) is the dissipated power of (a4) and verified by (1). The difference between $(a 2)$ and $(a 5)$, and between $(a 3)$ and $(a 6)$ state our improvement.

\section{(B) The natural approach improves the results given in $[30$ such as:}

The actual energy function for $(b 1)$ is

$$
\begin{aligned}
V(t, x, y) & =\frac{1}{2} y^{2}+\int_{0}^{x(t)}\left[p(t) g_{1}(s)\right. \\
& \left.+q(t) g_{2}(s)\right] d s
\end{aligned}
$$

where $d s=x^{\prime}(t) d t, p>0$ and $q>0$ are continuous functions on $[0, \infty), g_{1}, g_{2}$ are continuous functions on $\Re$, satisfying $\left(A_{1}\right)$ of [30].

Then, the time derivative of (b4) along the solutions of (b1) gives

$$
V^{\prime}(t)=-y^{2}<0 .
$$

In fact, the coefficient of $x^{\prime}$ in (b1) is 1 , and (b5) is confirmed by (1) due to the suitable tool. The comparisons between ( $b 2)$ and (b4), and between (b3) and (b5) give our improvement.

Further, the approach in this study also improves many results in the books [1] and 24] that based on Lyapunov approach. This list can be extended for the other related references that not cited here.

\section{Conclusion}

The energy of a system determines its behavior. In this context, this paper plays two important roles in the Lyapunov stability theory. First, it provides the construction of the energy function, which may obtain from the physical meaning of the given system. Second, it implies that the derivative of the energy function along the system trajectories is equal to the negative value of the 
dissipated power in the system. These further clarify the Lyapunov stability. Hence, one can nicely check the derivative of the energy function of a given physical system with (1). The proposed approach can be applicable to higher order differential systems. From now on, everyone involved in the subject will be able to find the same stability results for a system under consideration. We hope this present work will open new doors in the stability analysis of differential systems.

\section{References}

[1] Vidyasagar, M. (2002). Nonlinear System Analysis. Society for Industrial and Applied Mathematics, USA.

[2] Tongren, D. (2007). Approaches to the Qualitative Theory of Ordinary Differential Equations: Dynamical Systems and Nonlinear Oscillations. World Scientific, Peking, China.

[3] Meiss, J.D. (2007). Differential Dynamical Systems. Revised Edition. Society for Industrial and Applied Mathematics, USA.

[4] Yang, C., Sun, J., Zhang, Q., \& Ma, X. (2013). Lyapunov stability and strong passivity analysis for nonlinear descriptor systems. IEEE Transactions on Circuits and Systems I: Regular Papers, 60(4), 1003-1012.

[5] Platonov, A.V. (2020). Stability analysis for nonlinear mechanical systems with nonstationary potential forces. 15th International Conference on Stability and Oscillations of Nonlinear Control Systems (Pyatnitskiy's Conference) (STAB), Moscow, Russia.

[6] Ateş, M., \& Laribi, S. (2018). New results on the global asymptotic stability of certain nonlinear RLC circuits. Turkish Journal of Electrical Engineering and Computer Science, 26(1), 434-441.

[7] Zhang, L., \& Yu, L. (2013). Global asymptotic stability of certain third-order nonlinear differential equations. Mathematical Methods in the Applied Sciences, 36(14), 1845-1850.

[8] Sen, N. (2020). Stability analysis of electrical RLC circuit described by the CaputoLiouville generalized fractional derivative. Alexandria Engineering Journal, 59(4), 20832090.

[9] Nagamani, G., \& Radhika, T. (2016). Dissipativity and passivity analysis of markovian jump neural networks with two additive time varying delays. Neural Processing Letters, 44(2), 571-592.

[10] Nagamani, G., Radhika, T., \& Zhu, Q. (2017). An improved result on dissipativity and passivity analysis of markovian jump stochastic neural networks with two delay components. IEEE Transactions on Neural Networks and Learning Systems, 28(12), 3018-3031.

[11] Rao, M.R.M. (1981). Ordinary Differential Equations Theory and Applications. Edward Arnold, Inc., London, UK.

[12] Andreev, A., \& Peregudova, O. (2020). The direct Lyapunov method in the motion stabilization problems of robot manipulators. 15th International Conference on Stability and Oscillations of Nonlinear Control Systems (Pyatnitskiy's Conference) (STAB), Moscow, Russia.

[13] Nagamani, G., Soundararajan, G., Subramaniam, R., \& Azeem, M. (2020). Robust extended dissipativity analysis for Markovian jump discrete-time delayed stochastic singular neural networks. Neural Computing and Applications, 32(13), 9699-9712.

[14] Balasubramaniam P, \& Nagamani, G. (2011). Global robust passivity analysis for stochastic interval neural networks with interval time-varying delays and markovian jumping parameters. Journal of Optimization Theory and Applications, 149(1), 197-215.

[15] Nagamani, G., Radhika T., \& Balasubramaniam, P. (2015). A delay decomposition approach for robust dissipativity and passivity analysis of neutral-type neural networks with leakage time-varying delay. Complexity, 21(5), 248-264.

[16] Sastry, S. (1999). Nonlinear Systems, Analysis, Stability, and Control. Springer, New York, USA.

[17] Jeltsema, D., Ortega, R., \& Scherpen, J.M.A. (2003). A novel passivity property of nonlinear RLC circuits. Proceedings 4th Mathmod Sympositum (ARGESIM Report No. 24), Vienna, Austria, 845-853.

[18] Jeltsema, D., Ortega, R., \& Scherpen, J.M.A. (2003). On passivity and power-balance inequalities of nonlinear RLC circuits. IEEE Transactions on Circuits Systems I: Fundamental Theory and Applications, 50(9), 1174-1179.

[19] Ramirez, H.S., \& Lopez, E.M.N. (2000). On the passivity of general nonlinear systems. Proceedings of 14th Symposium on Mathematical Theory of Networks and Systems, Perpignan, France, 1-6.

[20] Zhu, F., Xia, M., \& Antsaklis, P.J. (2014). Passivity Analysis and Passivation of Interconnected Event-Triggered Feedback Systems Using Passivity Indices. Proceedings of the 
19th World Congress the International Federation of Automatic Control, Cape Town, South Africa, 24-29.

[21] Willems, J.C. (1972). Dissipative dynamical systems part I: General theory. Archive for Rotational Mechanics and Analysis, 45(5), 321-3519.

[22] Wang, J.L., Wu, H.N., Huang, T., Ren, S.Y., \& Wu, J. (2018). Passivity and output synchronization of complex dynamical networks with fixed and adaptive coupling strength. IEEE Transactions on Neural Networks and Learning Systems, 29(2), 364-376.

[23] Wang, J.L., Wu, H.N., Huang, T., Ren, S.Y., \& Wu, J. (2017). Passivity of directed and undirected complex dynamical networks with adaptive coupling weights. IEEE Transactions on Neural Networks and Learning Systems, 28(8), 1827-1839.

[24] Khalil, H.K. (2015). Nonlinear Control. Pearson Education, London, UK.

[25] Tunç, C., \& Tunç, E. (2007). On the asymptotic behavior of solutions of second order differential equations. Journal of The Franklin Institute, 344(5), 391-398.

[26] Lee, T.C., Tan, Y., \& Mareels, I. (2020). Detectability and uniform global asymptotic stability in switched nonlinear time-varying systems. IEEE Transactions on Automatic Control, 65(5), 2123-2138.

[27] Abate, A., Ahmed, D., Giacobbe, M., \& Peruffo, A. (2021). Formal synthesis of Lyapunov neural networks. IEEE Control Systems Letters, 5(3), 773-778.
[28] Chua, L.O., Desoer, C.A., \& Kuh, E.S. (1987). Linear and Nonlinear Circuits. McGraw-Hill, New York, USA.

[29] Dennis, D.Z., \& Michael, R.C. (2000). Advanced engineering mathematics. Jones and Bartlett Publisher, London, UK.

[30] Sugie, J., \& Amano, Y. (2004). Global asymptotic stability of nonautonomous systems of Lienard type. Journal of Mathematical Analysis and Applications, 289, 673-690.

Muzaffer Ates received his B.Sc. degree in Electrical and Electronics Engineering from METU, Turkey. Then he received his M.Sc. and PhD degrees in applied mathematics from Van Yuzuncu Yil University, Van, Turkey. Now, he is working at Electrical and Electronics Engineering Department in Van Yuzuncu Yil University. His research areas are nonlinear systems, mathematical control theory, circuit theory and Lyapunov stability theory.

(D) https://orcid.org/0000-0002-4394-0815

Nezir Kadah received his B.Sc. degree from Mersin University, Mersin, Turkey, in 2012 and his M.Sc. degree from Van Yuzuncu Yil University, Van, Turkey, in 2019. He is currently a PhD student of Electrical and Electronics Engineering at Adana Alparslan Turkes Science and Technology University (ATU), since 2019. He is also working at the department of Information Technology of ATU as the system and network administrator. His research areas are nonlinear systems, system identification, and control theory. (1) https://orcid.org/0000-0001-9320-1140

An International Journal of Optimization and Control: Theories \& Applications (http://ijocta.balikesir.edu.tr)

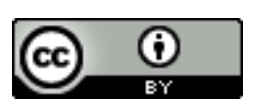

This work is licensed under a Creative Commons Attribution 4.0 International License. The authors retain ownership of the copyright for their article, but they allow anyone to download, reuse, reprint, modify, distribute, and/or copy articles in IJOCTA, so long as the original authors and source are credited. To see the complete license contents, please visit http://creativecommons.org/licenses/by/4.0/. 